\title{
Refill-Based Medication Use Quality Measures in Kidney Transplant Recipients: Examination of Proportion of Days Covered and Medication Possession Ratio
}

\author{
Brooke A. Hofmeyer, PharmD, MPH, BCPS; Kevin A. Look, PharmD, PhD; and David R. Hager, PharmD, BCPS
}

\begin{abstract}
BACKGROUND: The Pharmacy Quality Alliance's definition of proportion of days covered (PDC) and medication possession ratio (MPR) have not been examined as potential quality measures in the kidney transplant recipient population.
\end{abstract}

OBJECTIVES: To (a) describe the frequency distribution of MPR and PDC using mycophenolic acid products in a real-world kidney transplant recipient population and (b) evaluate associations between MPR and PDC with late (>90 days after transplantation) biopsy-proven acute rejection (BPAR). METHODS: This was a retrospective cohort study combining data from the Wisconsin Allograft Recipient Database with University of Wisconsin (UW) Health Specialty Pharmacy prescription claims and dispensing data from March 10, 2006, to June 30, 2012. Patients who met criteria for persistence filling mycophenolic acid prescriptions at UW Health Specialty Pharmacy in the first year following discharge from kidney transplantation surgery hospitalization were included. Patients were excluded if they were enrolled in a clinical trial, if they had BPAR within 90 days of transplantation, or if they did not have panel reactive antibody data available. PDC and MPR were calculated over 360 days after discharge, and multivariable analyses were performed to determine if there were associations between PDC or MPR with late BPAR within 3 years.

RESULTS: This study included 388 patients. The incidence of 3-year late BPAR was $5.1 \%(n=20)$. Characteristics of patients who experienced late BPAR were largely consistent with those who did not experience late BPAR, with the exception of number of hospital readmissions, which was higher among patients who experienced late BPAR. The frequency distribution of PDC and MPR exhibited a skewed left distribution, with a median PDC of 0.972 and a median MPR of 1.000 . Higher PDC was associated with lower odds of late $\mathrm{BPAR}(\mathrm{OR}=0.041,95 \% \mathrm{Cl}=0.004-0.417)$ in multivariable analysis, as was a higher MPR $(\mathrm{OR}=0.041,95 \% \mathrm{Cl}=0.004-0.419)$.

CONCLUSIONS: MPR and PDC may be calculated from data available to pharmacies and health plans, and each was associated with 3-year late BPAR among patients who did not experience early BPAR. However, the construct validity of these medication adherence measures requires further study.

J Manag Care Spec Pharm. 2018;24(4):367-72

Copyright $\odot 2018$, Academy of Managed Care Pharmacy. All rights reserved.

\section{What is already known about this subject}

Lifelong adherence to immunosuppressive medications following kidney transplantation is vital to prevent rejection of the allograft. Pharmacy prescription claims data have been used for the purpose of comparing health plans by national quality organizations in several chronic disease states, although this strategy has not been examined in kidney transplant recipients.

\section{What this study adds}

This study provides a description of medication possession ratio (MPR) and proportion of days covered (PDC), using mycophenolic acid products (mycophenolate mofetil or enteric-coated mycophenolate sodium) in a real-life kidney transplant recipient population.

MPR and PDC in the first year following kidney transplantation are associated with late biopsy-proven acute rejection within 3 years following kidney transplantation, yet the construct validity of these medication adherence measures requires further study.

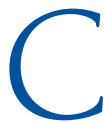
are following kidney transplantation requires lifelong adherence to immunosuppressant medications. Nonadherence to these medications after kidney transplantation is a common, dynamic, and modifiable risk factor for costly acute rejection episodes and allograft loss secondary to chronic, active antibody-mediated rejection. ${ }^{1,2}$ Health system attributes may influence medication adherence after kidney transplantation, as evidenced by differential adherence rates observed in American and European transplant recipients. ${ }^{3,4}$ However, there is a lack of literature describing medication adherence quality measures, such as those endorsed by national quality organizations, for the purpose of comparing health plans and specialty pharmacies serving kidney transplant recipient populations. ${ }^{5}$

The Pharmacy Quality Alliance has developed, tested, and endorsed measures intended to estimate medication adherence in many disease states, using data available to prescription drug plans and closed pharmacy systems. ${ }^{6,7}$ Proportion of days covered (PDC) and medication possession ratio (MPR) can be used to track medication adherence from one year to the next. ${ }^{8}$ PDC is preferred over MPR, since it is less likely to overestimate adherence because of prospective adjustment for overlapping days supplied. ${ }^{6,9}$ The Centers for Medicare \& Medicaid Services used the PDC adherence measures endorsed by the National Quality Forum for statins, renin angiotensin system antagonists, and oral diabetes medications in their Part D 2016 star ratings, many of which correlate with Part $C$ intermediate outcomes. ${ }^{10}$ A PDC threshold of $\geq 0.800(\geq 80 \%)$ is typically selected to dichotomize medication adherence in disease states 
such as schizophrenia, diabetes, hypertension, congestive heart failure, and hyperlipidemia based on hospitalization predictive value. ${ }^{11,12}$ However, neither PDC nor MPR has been applied to characterize use of oral immunosuppressant medication in a real-world kidney transplant recipient population, so distributions of adherence rates using PDC and MPR in this distinct clinical arena are unknown.

The objectives of this study were to (a) describe the frequency distribution of MPR and the PDC using mycophenolic acid product-dispensing records in a real-world kidney transplant recipient population, and (b) determine whether there is an association between MPR or PDC in the first year following kidney transplantation and the 3-year incidence of late biopsyproven acute rejection (BPAR), adjusting for a limited number of patient characteristics in multivariable analysis. Late BPAR was chosen as the primary outcome because it is commonly associated with nonadherence to immunosuppressant medications, responds poorly to treatment, and frequently leads to accelerated allograft loss. ${ }^{2,13,14}$ The results of this study were interpreted in the context of the unique epidemiology of kidney transplant recipient rehospitalization and immunosuppression standards of care following kidney transplantation.

\section{Methods}

\section{Study Population and Databases}

Data from the Wisconsin Allograft Recipient Database (WisARD) were combined with prescription claims data from the University of Wisconsin (UW) Health Specialty Pharmacy. WisARD contains prospectively validated data on demographics, transplant- and nontransplant-related outcomes, treatment, and laboratory values for all patients who had a solid organ transplantation procedure at the UW Hospital from 1980 to the present. Patients with a kidney transplantation surgery discharge between March 10, 2006, and June 20, 2012 ( $\mathrm{n}=1,640$ ) were included based on availability of data.

Patients were excluded if they experienced BPAR within 90 days of transplantation, since this study was neither designed nor powered to evaluate factors that would predispose a patient to acute cellular or antibody-mediated rejection within this early time frame after transplantation. ${ }^{14}$ BPAR within 90 days would also have limited the MPR and PDC observation period. Patients were also excluded if they were enrolled in a clinical trial and if panel reactive antibody (PRA) data were not available at the time of transplantation.

Mycophenolic acid products (mycophenolate mofetil and enteric-coated mycophenolate sodium) were chosen for measurement, since they are expected to be subject to fewer dose adjustments than calcineurin inhibitors and corticosteroids in the first year following transplantation, and all patients were expected to remain on mycophenolic acid throughout the first postoperative year as standard of care at the study institution. According to the recommendations of the Pharmacy Quality Alliance, the MPR and PDC denominator should include the patients who were continuously enrolled in the pharmacy during the observation period. Therefore, persistence on mycophenolic acid was defined as at least 1 mycophenolic acid prescription within 15 days of discharge, 1 mycophenolic acid prescription within 100 days of day 360 (to account for 90-day supplies), and at least 3 prescriptions within the observation period.

The primary outcome was 3-year late BPAR. All subjects were observed for a period of 3 years following kidney transplantation, regardless of their transplantation date. We selected covariates among available data a priori, by those most associated with the primary outcome. The UW Health Sciences Minimal Risk Institutional Review Board approved this study.

\section{Definition of Medication Possession Ratio}

Medication possession ratio (MPR) was defined as the sum of days supplied for each prescription dispensed between the date of discharge and 360 days following discharge, divided by 360 days. The days supplied for the last fill before the end of the observation period was truncated by the number of days between the fill and the last day of the study period. The maximum MPR was limited to 1.000 (100\%).

\section{Definition of Proportion of Days Covered}

Proportion of days covered (PDC) was defined using a microarray approach using days supplied as described by Leslie for the 2007 SAS Global Forum. ${ }^{15}$ Briefly, an array was developed to account for each of 360 days following each patient's date of discharge. The days supplied by each mycophenolic acid prescription fill prospectively populated each day in the array with a value of 1 to indicate that medication was available that day or 0 to indicate no availability. Overlapping days supplied were credited forward to the day with the next 0 . As with MPR, the days supplied for the last prescription fill within the observation period was truncated, and the maximum PDC was limited to $1.000(100 \%)$.

\section{Statistical Analysis}

Descriptive statistics and a multivariable analysis were conducted using continuous data for MPR and PDC in accordance with the International Society for Pharmacoeconomics and Outcomes Research because of the lack of empiric evidence for a cutpoint or "threshold" in this population. ${ }^{16}$ Logistic regression was used to conduct the multivariable analyses, using incidence of late $\operatorname{BPAR}(1,0)$ as the primary outcome. All statistical analyses were performed in Stata 14 (StataCorp, College Station, TX).

\section{Results}

A total of 388 patients were included in the analysis. Of the initial 1,640 patients, 238 were excluded for BPAR within the first 90 days after transplantation; 45 were then excluded for 
Refill-Based Medication Use Quality Measures in Kidney Transplant Recipients:

Examination of Proportion of Days Covered and Medication Possession Ratio

\begin{tabular}{|c|c|c|c|c|}
\hline Demographics & No Late BPAR $(\mathrm{n}=368)$ & Late BPAR $(n=20)$ & $P$ Value & Test Used \\
\hline Age, mean (SD) & $50.3(13.3)$ & $49.9(13.9)$ & 0.960 & Wilcoxon rank-sum \\
\hline Female, n (\%) & 141 (38.3) & $6(30.0)$ & 0.640 & Fisher exact \\
\hline \multicolumn{5}{|l|}{ Race, n (\%) } \\
\hline White & $302(82.1)$ & $15(75.0)$ & \multirow{3}{*}{0.500} & \multirow{3}{*}{ Fisher exact } \\
\hline Black & $35 \quad(9.5)$ & $2(10.0)$ & & \\
\hline Other & $31 \quad(8.4)$ & $3(15.0)$ & & \\
\hline ESRD due to diabetes, $\mathrm{n}(\%)$ & $71 \quad(19.3)$ & $2(10.0)$ & 0.390 & Fisher exact \\
\hline Prior transplantations count, mean (SD) & $1.2 \quad(0.5)$ & $1.2 \quad(0.7)$ & 0.490 & Wilcoxon rank-sum \\
\hline \multicolumn{5}{|l|}{ Donor type, $\mathrm{n}(\%)$} \\
\hline Live & $136(37.0)$ & $8 \quad(40.0)$ & \multirow{3}{*}{0.770} & \multirow{3}{*}{ Fisher exact } \\
\hline Brain death & $147 \quad(40.0)$ & $9 \quad(45.0)$ & & \\
\hline Cardiac death & $85(23.1)$ & $3(15.0)$ & & \\
\hline PRA, mean (SD) & $4.6 \quad(15.4)$ & $5.5(12.4)$ & 0.230 & Wilcoxon rank-sum \\
\hline Hospital readmissions count, mean (SD) & $0.4 \quad(0.9)$ & $1.0 \quad(1.2)$ & $<0.001$ & Wilcoxon rank-sum \\
\hline
\end{tabular}

enrollment in a clinical trial; 251 of the remaining were excluded because they did not fill a mycophenolic acid product at a UW pharmacy within 15 days of discharge; and an additional 712 did not meet criteria for persistence filling at a UW pharmacy for the entire 360-day observation period. Finally, 6 patients were excluded for missing PRA data.

Distributions of MPR and PDC exhibited a skewed left distribution, with a median PDC of 0.972 (interquartile range $[\mathrm{IQR}]=0.128)$ and a median MPR of $1.000(\mathrm{IQR}=0.108)$. The mean PDC was 0.907 (standard deviation $[S D]=0.146$ ) and mean MPR was 0.920 ( $S D=0.143)$. A total of 139 patients had PDC capped at 1.000 , and 202 patients had MPR capped at 1.000. There were 34 cases per 100 patients per year with a $\mathrm{PDC}<1.000$ and 28 cases per 100 patients per year with an $\mathrm{MPR}<1.000$.

The incidence of late ( $>90$ days after transplantation) BPAR within 3 years after transplantation in this cohort was $5.1 \%(\mathrm{n}=20)$, with a mean onset of 266.8 days ( $\mathrm{SD}=186.7$ days, range $=103-806$ days) after kidney transplantation. Characteristics of patients who experienced late BPAR were largely consistent with those who did not experience late BPAR on the basis of age $(P=0.96)$, sex $(P=0.64)$, race $(P=0.50)$, diabetes as the cause of native kidney disease $(P=0.39)$, previous solid organ transplantation $(P=0.49)$, donor type $(P=0.77)$, and PRA $(P=0.23$; Table 1). However, patients with late BPAR had a mean first year hospital readmission count of $1.0(\mathrm{SD}=1.2)$, whereas patients without late BPAR had a mean hospital readmission count of 0.4 ( $\mathrm{SD}=0.9 ; P<0.001$; Table 1). Both MPR (odds ratio $[\mathrm{OR}]=0.041 ; 95 \%$ confidence interval $[\mathrm{CI}]=0.005$ $0.334)$ and $\mathrm{PDC}(\mathrm{OR}=0.42 ; 95 \% \mathrm{CI}=0.005-0.341)$ were associated with late BPAR in bivariate analyses. In the multivariable models, MPR $(\mathrm{OR}=0.041 ; 95 \% \mathrm{CI}=0.004-0.419 ; \mathrm{P}<0.007)$ and $\mathrm{PDC}(\mathrm{OR}=0.041 ; 95 \% \mathrm{CI}=0.004-0.417 ; \mathrm{P}<0.007)$ remained similarly associated with late BPAR (Table 2). Excluding hospital readmissions count did not have a significant effect on MPR (OR $=0.030 ; 95 \% \mathrm{CI}=0.003-0.283 ; \mathrm{P}=0.002)$ or $\mathrm{PDC}$ $(\mathrm{OR}=0.031 ; 95 \% \mathrm{CI}=0.003-0.284 ; \mathrm{P}=0.002)$.

\section{Discussion}

Successful kidney transplantations are cost saving to society, while the collective cost of rejection treatments and allograft failures ranges from $\$ 15$ to $\$ 100$ million per year. ${ }^{17,18}$ Associations between medication nonadherence, BPAR, and graft loss have been previously demonstrated., ${ }^{1,2,19-21}$

In these multivariate analyses, each cumulative percentage point of mycophenolic acid MPR and PDC in the first year following kidney transplantation discharge was associated with considerably decreased odds of 3-year late BPAR (Table 2). Medication adherence is important for all organ transplant recipients, and while modifiable, interventions to improve medication adherence can be resource intensive. ${ }^{22}$ The constructs of MPR and PDC based solely on mycophenolic acid pharmacy records could be used by health plans and pharmacies with or without access to data other than the prescription records, in order to identify patients who may require closer monitoring. ${ }^{23}$ In this context, an exercise in triangulation could be used to collect information from multiple sources and disciplines concerning specific patients, as has been examined in another population that demands a high degree of medication adherence-in patients with human immunodeficiency virus-in order to personalize pharmaceutical care services. ${ }^{24,25}$ Without direct interaction with patients and providers, potential users of these measures should consider the lack of evidence for the utility of a specific MPR or PDC threshold, as well as the multiple constructs that PDC and MPR may actually measure. 
Refill-Based Medication Use Quality Measures in Kidney Transplant Recipients:

Examination of Proportion of Days Covered and Medication Possession Ratio

TABLE 2 Multivariable Analyses of BPAR with MPR or PDC in Model

\begin{tabular}{|c|c|c|c|c|}
\hline \multirow[b]{2}{*}{ Variable (Comparator) } & \multicolumn{2}{|c|}{$\begin{array}{l}\text { Adjusted MPR Model } \\
\text { c-statistic: } 0.780\end{array}$} & \multicolumn{2}{|c|}{$\begin{array}{l}\text { Adjusted PDC Model } \\
\text { c-statistic: } 0.776\end{array}$} \\
\hline & OR $(95 \% \mathrm{CI})$ & $P$ Value & OR $(95 \% \mathrm{CI})$ & $P$ Value \\
\hline MPR or PDC, continuous & $0.041 \quad(0.004-0.419)$ & $<0.007$ & $0.041 \quad(0.004-0.417)$ & $<0.007$ \\
\hline Age, continuous & $1.000 \quad(0.965-1.037)$ & 0.978 & $1.000 \quad(0.965-1.036)$ & 0.981 \\
\hline Female (male) & $0.610 \quad(0.215-1.731)$ & 0.353 & $0.593 \quad(0.208-1.686)$ & 0.327 \\
\hline Black (white) race & $1.168 \quad(0.239-5.720)$ & 0.848 & $1.160 \quad(0.236-5.680)$ & 0.885 \\
\hline Other (white) race & $2.481 \quad(0.604-10.202)$ & 0.208 & $2.552 \quad(0.622-10.480)$ & 0.194 \\
\hline Diabetes (other) primary kidney disease & $0.426 \quad(0.088-2.070)$ & 0.290 & $0.433 \quad(0.089-2.103)$ & 0.299 \\
\hline Prior transplantations count, continuous & $0.793 \quad(0.242-2.603)$ & 0.703 & $0.791 \quad(0.241-2.601)$ & 0.700 \\
\hline Brain death (living) donor & $1.130 \quad(0.384-3.322)$ & 0.824 & $1.111 \quad(0.379-3.255)$ & 0.848 \\
\hline Cardiac death (living) donor & $0.380 \quad(0.081-1.771)$ & 0.217 & $0.377 \quad(0.081-1.762)$ & 0.215 \\
\hline Panel reactive antibodies \%, continuous & $1.005 \quad(0.973-1.039)$ & 0.746 & $1.006 \quad(0.974-1.039)$ & 0.718 \\
\hline Hospital readmissions count, continuous & $1.502 \quad(1.071-2.106)$ & 0.018 & $1.502 \quad(1.071-2.105)$ & 0.018 \\
\hline
\end{tabular}

Deviation from the prescribed regimen sufficient to influence adverse outcomes, known as "clinical nonadherence," was the preferred definition of nonadherence by experts in the field of kidney transplantation who convened at a 2009 consensus conference. ${ }^{17}$ However, risk of adverse clinical outcomes with any particular degree of medication nonadherence in this field remains highly patient specific. As in many fields, current standards of care in transplantation immunosuppression do not yet reflect the ideals of personalized medicine, making it difficult to assess the influence of nonadherence to medications on the course of this chronic condition. ${ }^{26,27}$ Treatments for late antibody-mediated and mixed rejections are expensive, not consistently effective, and frequently toxic. ${ }^{13,28-31}$ Late antibodymediated rejections and mixed rejections are emerging as leading causes of allograft loss, limiting improvements in long-term survival after kidney transplantation; complex immunologic processes underpin these cases, and medication adherence seems to matter. ${ }^{2,32,33}$ The effects of immunologic compatibility of donor-recipient pairs in the context of dynamic cellular and molecular signaling, subclinical nonadherence, and iatrogenic under-immunosuppression remain to be fully characterized. $^{32,34,35}$ Immunosuppression personalization and promotion of adherence among patients at highest risk for late BPAR may therefore be key to improving outcomes in the future. ${ }^{36}$

The frequency distributions of MPR and PDC observed in this cohort were skewed further left when compared with other chronic disease states in which the MPR and PDC denominators have been similarly defined. ${ }^{37}$ This finding may suggest either a greater degree of medication adherence or simply more frequent dose changes and trips to the pharmacy among kidney transplant recipients. ${ }^{9}$ Using the most inclusive threshold of $<1.000$ to define possible nonadherence, there were 28 (MPR) to 34 (PDC) cases of nonadherence per 100 patients per year, similar to the incidence described in a 2007 meta-analysis of nonadherence in kidney transplant recipients of 36 cases of nonadherence per 100 patients per year. ${ }^{38}$ The PDC threshold of $<0.800$ (80\%) commonly selected for its hospitalization predictive value in chronic disease states has not been validated among kidney transplant recipients and may not have much utility in this setting. Future studies are needed to determine whether a higher threshold would be useful for predicting outcomes meaningful to the kidney transplant recipient population, such as hospitalization predictive value, allograft loss, or survival.

\section{Limitations}

This analysis was limited by the lack of data on days spent hospitalized and inpatient medication use, since these data were not available in WisARD. Patients in this analysis were therefore assumed to be users of outpatient medications each day during the observation period. The Centers for Medicare \& Medicaid Services have performed adjustments of PDC for inpatient, hospice, and skilled nursing facility stays and found little effect on the PDC measurement. ${ }^{39}$ However, hospitalization after kidney transplantation is prevalent and highly associated with recipient age; in 1 single center study, $4.2 \%-8.6 \%$ of patients spent 120 or more excess days in the hospital in the first year after kidney transplantation. ${ }^{40,41}$ Moreover, $51 \%$ of patients with allograft rejection may require prolonged hospital stays. ${ }^{42}$ It is plausible that hospitalization may mediate the observed association between MPR and PDC with late BPAR in this analysis, even though the observation period for the primary outcome was 3 times as long as the observation period for the primary independent variables (i.e., for MPR and PDC). Additional information about hospitalization may be available to health plans and integrated health care systems that would be interested in using either MPR or PDC to estimate outpatient immunosuppressant medication use. Future research should investigate the 


\section{Refill-Based Medication Use Quality Measures in Kidney Transplant Recipients: Examination of Proportion of Days Covered and Medication Possession Ratio}

influence of inpatient hospitalization within the observation period and the prospective hospitalization predictive value of MPR and PDC to further develop their potential utility in quality assessment and performance improvement.

While most kidney transplant recipients will be initiated on a mycophenolic acid product after transplantation, some will be converted to another antiproliferative medication such as azathioprine. Frequent dose changes may influence MPR and PDC in the early period after transplantation if using refill data for medications such as calcineurin inhibitors. On the flip side, monthly belatacept infusions would be relatively straightforward to monitor. Taking into account the variety of regimens that may be used in solid organ transplantation, a multiclass approach may need to be taken. ${ }^{43}$ For example, Pinsky et al. (2009) reported a modified MPR of 0.896 during the first year after kidney transplantation using a multidrug approach. ${ }^{44}$

Finally, the generalizability of these data may also be limited because patients who choose to continue to fill their mycophenolic acid prescriptions at a health system pharmacy may not be representative of the overall population transplanted at this single center or at any other transplant center. There was limited racial heterogeneity among participants and fewer patients with end-stage renal disease secondary to diabetes than in many populations (Table 1). While data on marriage status were unknown, there was a high representation of recipients of living donor kidney transplantations (Table 1). It is not uncommon for living donors to be spouses who, in turn, may help improve medication adherence.

\section{Conclusions}

In the first year following kidney transplantation discharge, MPR and PDC of mycophenolic acid product prescriptions were each associated with late BPAR within 3 years following kidney transplantation. However, their utility as medication use quality measures is questionable because of their limited distribution even before adjustment for hospitalization. Further research should consider the construct validity of these medication adherence measures in a kidney transplant recipient population, as well as investigating censoring and longterm temporal relationships between claims-based medication adherence measurements and hospitalizations, kidney allograft loss, and survival.

\section{Authors}

BROOKE A. HOFMEYER, PharmD, MPH, BCPS, Department of Pharmaceutical Care, University of Iowa Hospitals and Clinics, Iowa City; KEVIN A. LOOK, PharmD, PhD, University of Wisconsin School of Pharmacy, Madison; and DAVID R. HAGER, PharmD, BCPS, Department of Pharmacy, University of Wisconsin Health, Madison.

AUTHOR CORRESPONDENCE: Brooke A. Hofmeyer, PharmD, MPH, BCPS, 200 Hawkins Dr., Iowa City, IA 52242.

E-mail: brooke-hofmeyer@uiowa.edu.

\section{DISCLOSURES}

This study was not funded. The authors report no conflicts of interest and no relevant financial interests related to the products or services discussed in this article.

Study concept and design were contributed by Hofmeyer, along with Look and Hager. Hager took the lead in data collection, along with the other authors. Data interpretation was performed by Look, along with the other authors. The manuscript was primarily written by Hofmeyer, assisted by Look and Hager, and revised by all of the authors.

\section{REFERENCES}

1. Butler JA, Roderick P, Mullee M, Mason JC, Peveler RC. Frequency and impact of nonadherence to immunosuppressants after renal transplantation: a systematic review. Transplantation. 2004;77(5):769-76.

2. Sellares J, de Freitas DG, Mengel M, et al. Understanding the causes of kidney transplant failure: the dominant role of antibody-mediated rejection and nonadherence. Am J Transplant. 2012;12(2):388-99.

3. Denhaerynck K, Desmyttere A, Dobbels F, et al. Nonadherence with immunosuppressive drugs: U.S. compared with European kidney transplant recipients. Prog Transplant. 2006;16(3):206-14

4. Desai V, Nau D, Conklin M, Heaton PC. Impact of environmental factors on differences in quality of medication use: an insight for the Medicare star rating system. J Manag Care Spec Pharm. 2016;22(7):779-86. Available at: https://www.jmcp.org/doi/10.18553/jmcp.2016.22.7.779.

5. Centers for Medicare $\&$ Medicaid Services. Measures Inventory Tool. 2018. Updated October 15, 2017. Available at: https://cmit.cms.gov. Accessed March 12, 2018.

6. Nau D. Proportion of days covered (PDC) as a preferred method of measuring medication adherence. Pharmacy Quality Alliance. Available at: http://www.pqaalliance.org/images/uploads/files/PQA\%20PDC\%20vs\%20 \%20MPR.pdf. Accessed February 27, 2018.

7. Nau DP. Measuring pharmacy quality. J Am Pharm Assoc (2003). 2009:49(2):154-63

8. Saundankar V, Peng X, Fu H, et al. Predictors of change in adherence status from 1 year to the next among patients with type 2 diabetes mellitus on oral antidiabetes drugs. J Manag Care Spec Pharm. 2016;22(5):467-82. Available at: https://www.jmcp.org/doi/10.18553/jmcp.2016.22.5.467.

9. Martin BC, Wiley-Exley EK, Richards S, Domino ME, Carey TS, Sleath BL. Contrasting measures of adherence with simple drug use, medication switching, and therapeutic duplication. Ann Pharmacother. 2009;43(1):36-44.

10. Ta JT, Erickson SC, Qiu WA, Patel BV. Is there a relationship between Part D medication adherence and Part C intermediate outcomes star ratings measures? J Manag Care Spec Pharm. 2016;22(7):787-95. Available at: https:// www.jmcp.org/doi/10.18553/jmcp.2016.22.7.787.

11. Osterberg L, Blaschke T. Adherence to medication. New Engl J Med. 2005;353(5):487-97

12. Karve S, Cleves MA, Helm M, Hudson TJ, West DS, Martin BC. Good and poor adherence: optimal cut-point for adherence measures using administrative claims data. Cur Med Res Opin. 2009;25(9):2303-10.

13. Redfield RR, Ellis TM, Zhong W, et al. Current outcomes of chronic active antibody mediated rejection-a large single center retrospective review using the updated BANFF 2013 criteria. Hum Immunol. 2016;77(4):346-52.

14. Dorje C, Midtvedt K, Holdaas H, et al. Early versus late acute antibodymediated rejection in renal transplant recipients. Transplantation. 2013; 96(1):79-84

15. Leslie RS. Using arrays to calculate medication utilization. Presented at: SAS Global Forum 2007; April 16-19, 2007; Orlando, FL. Available at: http://www2.sas.com/proceedings/forum2007/043-2007.pdf. Accessed February 27, 2018. 


\section{Refill-Based Medication Use Quality Measures in Kidney Transplant Recipients: Examination of Proportion of Days Covered and Medication Possession Ratio}

16. Peterson AM, Nau DP, Cramer JA, Benner J, Gwadry-Sridhar F, Nichol M. A checklist for medication compliance and persistence studies using retrospective databases. Value Health. 2007;10(1):3-12.

17. Fine RN, Becker Y, De Geest S, et al. Nonadherence consensus conference summary report. Am J Transplant. 2009;9(1):35-41.

18. Winkelmayer WC, Weinstein MC, Mittleman MA, Glynn RJ, Pliskin JS. Health economic evaluations: the special case of end-stage renal disease treatment. Med Decis Making. 2002;22(5):417-30.

19. Denhaerynck K, Dobbels F, Cleemput I, et al. Prevalence, consequences, and determinants of nonadherence in adult renal transplant patients: a literature review. Transpl Int. 2005;18(10):1121-33.

20. Vlaminck H, Maes B, Evers G, et al. Prospective study on late consequences of subclinical non-compliance with immunosuppressive therapy in renal transplant patients. Am J Transplant. 2004:4(9):1509-13.

21. Wiebe C, Gibson IW, Blydt-Hansen TD, et al. Evolution and clinical pathologic correlations of de novo donor-specific HLA antibody post kidney transplant. Am J Transplant. 2012;12(5):1157-67.

22. Joost R, Dorje F, Schwitulla J, Eckardt KU, Hugo C. Intensified pharmaceutical care is improving immunosuppressive medication adherence in kidney transplant recipients during the first post-transplant year: a quasiexperimental study. Nephrol Dial Transplant. 2014;29(8):1597-607.

23. Bagwell A, Kelley T, Carver A, Lee JB, Newman B. Advancing patient care through specialty pharmacy services in an academic health system. J Manag Care Spec Pharm. 2017;23(8):815-20. Available at: https://www.jmcp. org/doi/10.18553/jmcp.2017.23.8.815

24. Liu H, Golin CE, Miller LG, et al. A comparison study of multiple measures of adherence to HIV protease inhibitors. Ann Intern Med. 2001;134(10):968-77.

25. Turner BJ, Hecht FM. Improving on a coin toss to predict patient adherence to medications. Ann Intern Med. 2001;134(10):1004-06.

26. Karagiannis TC. The timeless influence of Hippocratic ideals on diet, salicytates and personalized medicine. Hell J Nucl Med. 2014;17(1):2-6.

27. Schork NJ. Personalized medicine: time for one-person trials. Nature 2015;520(7549):609-11

28. Muduma G, Odeyemi I, Smith-Palmer J, Pollock RF. Review of the clinical and economic burden of antibody-mediated rejection in renal transplant recipients. Adv Ther. 2016;33(3):345-56

29. Sautenet B, Blancho G, Buchler M, et al. One-year results of the effects of rituximab on acute antibody-mediated rejection in renal transplantation: RITUX ERAH, a multicenter double-blind randomized placebo-controlled trial. Transplantation. 2016;100(2):391-99.

30. Smith RN, Malik F, Goes N, et al. Partial therapeutic response to rituximab for the treatment of chronic alloantibody mediated rejection of kidney allografts. Transpl Immunol. 2012;27(2-3):107-13.
31. Djamali A, Muth BL, Torrealba J, et al. Bortezomib as a rescue therapy for hyperacute and multi-drug resistant mixed acute rejection after kidney transplantation. Clin Transpl. 2009:485-90.

32. Nankivell BJ, Alexander SI. Rejection of the kidney allograft. New Engl J Med. 2010;363(15):1451-62.

33. Walsh RC, Brailey P, Girnita A, et al. Early and late acute antibodymediated rejection differ immunologically and in response to proteasome inhibition. Transplantation. 2011;91(11):1218-26.

34. Wiebe C, Nevins TE, Robiner WN, Thomas W, Matas AJ, Nickerson PW. The synergistic effect of class II HLA epitope-mismatch and nonadherence on acute rejection and graft survival. Am J Transplant. 2015;15(8):2197-202.

35. Duquesnoy RJ. The antibody response to HLA mismatch: putting together the pieces of a puzzle. Am J Transplant. 2015;15(12):3019-20.

36. Sorensen TD, Pestka DL, Brummel AR, Rehrauer DJ, Ekstrand MJ. Seeing the forest through the trees: improving adherence alone will not optimize medication use. J Manag Care Spec Pharm. 2016;22(5):598-604. Available at: https://www.jmcp.org/doi/10.18553/jmcp.2016.22.5.598.

37. Yeaw J, Benner JS, Walt JG, Sian S, Smith DB. Comparing adherence and persistence across 6 chronic medication classes. J Manag Care Pharm. 2009;15(9):728-40. Available at: https://www.jmcp.org/doi/10.18553/ jmcp.2009.15.9.728.

38. Dew MA, DiMartini AF, De Vito Dabbs A, et al. Rates and risk factors for nonadherence to the medical regimen after adult solid organ transplantation. Transplantation. 2007;83(7):858-73.

39. Centers for Medicare \& Medicaid Services. Medicare 2017 Part C \& D star ratings technical notes. First plan preview. Draft. August 3, 2016. Available at: https://www.cms.gov/Medicare/Prescription-Drug-Coverage/ PrescriptionDrugCovGenIn/Downloads/2017_Technical_Notes_preview_1_2016_08_03.pdf. Accessed February 27, 2018.

40. Grams ME, McAdams Demarco MA, Kucirka LM, Segev DL. Recipient age and time spent hospitalized in the year before and after kidney transplantation. Transplantation. 2012;94(7):750-56.

41. Naderi M, Aslani J, Hashemi M, Assari S, Amini M, Pourfarziani V. Prolonged rehospitalizations following renal transplantation: causes, risk factors, and outcomes. Transplant Proc. 2007;39(4):978-80.

42. Pourfarziani V, Nourbala MH, Azizabadi Farahani M, Moghani Lankarani M, Assari S. Costs and length of hospitalizations following kidney transplantation. Iran J Kidney Dis. 2009;3(2):103-08.

43. Choudhry NK, Shrank WH, Levin RL, et al. Measuring concurrent adherence to multiple related medications. Am J Manag Care. 2009;15(7):457-64.

44. Pinsky BW, Takemoto SK, Lentine KL, Burroughs TE, Schnitzler MA, Salvalaggio PR. Transplant outcomes and economic costs associated with patient noncompliance to immunosuppression. Am J Transplant. 2009;9(11):2597-606 